Research Paper

\title{
MicroRNA expression profile of Lacrimal Glands in rabbit autoimmune dacryoadenitis model
}

\author{
Bo Liuㅍ, Lu Zhao#, Yankai Wei², Sisi Chen², Lingzhai Bian², Di Guo², Min Gao², Hong Nian² ${ }^{\bowtie}$ \\ 1. Department of Neurosurgery, Tianjin Medical University General Hospital, Tianjin, China \\ 2. Tianjin Key Laboratory of Retinal Functions and Diseases; Tianjin International Joint Research and Development Centre of Ophthalmology and Vision Science; Eye \\ Institute and School of Optometry, Tianjin Medical University Eye Hospital, Tianjin, China. \\ \#These authors contributed equally to this work.
}

$\triangle$ Corresponding author: Hong Nian, Ph.D., Tianjin Key Laboratory of Retinal Functions and Diseases; Tianjin International Joint Research and Development Centre of Ophthalmology and Vision Science; Eye Institute and School of Optometry, Tianjin Medical University Eye Hospital, Tianjin 300384, China; E-mail: nianhong@tmu.edu.cn.

(C) The author(s). This is an open access article distributed under the terms of the Creative Commons Attribution License (https://creativecommons.org/licenses/by/4.0/). See http://ivyspring.com/terms for full terms and conditions.

Received: 2020.07.03; Accepted: 2020.09.14; Published: 2020.10.16

\begin{abstract}
Purpose: To identify the differential expression of microRNAs (miRs) and the related gene networks and signal pathways in lacrimal glands (LGs) of rabbit autoimmune dacryoadenitis.

Methods: Autoimmune dacryoadenitis in rabbits was induced by transferring activated peripheral blood lymphocytes (PBLs). The LGs of normal and model group rabbits were collected for small RNA sequencing. The most differentially expressed miRs were validated by quantitative real time-polymerase chain reaction (qRT-PCR). Further, bioinformatics analysis including target gene prediction, Gene Ontology (GO) terms and Kyoto Encyclopedia of Genes and Genomes (KEGG) pathway enrichment analyses were performed.

Results: A total of 15 miRs were differentially expressed in the LGs of rabbit autoimmune dacryoadenitis relative to normal controls. GO and KEGG analysis revealed that most target genes of these dysregulated miRs were implicated in MAPK signaling pathway.

Conclusion: Our results showed for the first time the differentially expressed miRs and the related pathways involved in the pathogenesis of rabbit autoimmune dacryoadenitis. These results may contribute to elucidating molecular pathogenesis of Sjögren's syndrome (SS) dry eye.
\end{abstract}

Key words: Small RNA sequencing; autoimmune dacryoadenitis; Sjögren's syndrome dry eye; MicroRNAs

\section{Introduction}

Sjögren's syndrome (SS) dry eye is a chronic ocular autoimmune disease characterized by ocular inflammation and dysfunction in LGs, which may ultimately result in visual impairment $[1,2]$. SS dry eye is considered to be mediated by multiple factors including genetic, environmental and epigenetic factors [3]. Although susceptibility to SS dry eye is in part genetic, and epigenetic modulation plays an important role in the development of various ocular conditions [4,5], studies investigating the epigenetic factors that contribute to the SS dry eye are relatively scarce.

To explore the detailed mechanisms of SS dry eye, several animal models have been developed [6]. Relative to other animal models, rabbit autoimmune dacryoadenitis, induced by injection of activated autologous PBLs, shares many common features with human SS [7]. Moreover, the clinical features and LG histopathology of this autoadoptively transferred dacryoadenitis can persist for at least 6 months, indicating a chronic feature matching the chronic features of SS and often lacking in induced or genetically determined rodent dry eye models [8].

microRNAs (miRs), a class of small singlestranded, non-coding RNAs, are highly conserved key regulators of various autoimmune processes and can epigenetically regulate gene expression at post-transcription level through mRNA degradation or protein synthesis inhibition [9, 10]. Recently, increasing evidences from clinical researches in 
patients and experimental animal models have shown that the dysregulation of miRs was closely related to the development of autoimmune diseases, including SS dry eye [11, 12]. miRs have been shown to be differentially expressed in the tears, peripheral blood mononuclear cells (PBMCs) and salivary glands from SS patients [13-15]. For example, Shi et al. demonstrated that the expression of miR-146a is upregulated and miR-155 is downregulated in PBMCs of patients with SS, and the expression levels of these miRs are correlated with the visual analog scale scores for dry eye [16]. However, little is known about the miRs expression profile in LGs of SS dry eye [17].

In this study, we characterized the differential expression profile of miRs in LGs of rabbit autoimmune dacryoadenitis relative to normal ones and predicted their potential roles using bioinformatics methods. Our studies may provide clues to develop new clinical intervention strategies of SS dry eye.

\section{Materials and Methods}

\section{Rabbit autoimmune dacryoadenitis model}

Adult female New Zealand white rabbits (3.5-4 $\mathrm{kg}$ ) were purchased from Vital River (Beijing, China) and were raised under pathogen-free conditions at Tianjin Medical University Eye hospital. The study was performed in accordance with the ARVO Statement for Use of Animals in Ophthalmic and Vision Research and approved by Institutional Animal Care and Use Committee of Tianjin Medical University.

All rabbits were subjected to a standardized ocular examination before the experimentation, and those with any pre-existing eye defects were excluded. Autoimmune dacryoadenitis was induced as introduced previously [18]. Briefly, the left inferior LGs and peripheral blood were collected for isolating purified LG epithelial cells (pLGECs) and PBLs. After two days of culture, the irradiated pLGECs were co-cultured with autologous PBLs in a 1:1 ratio for activating PBLs. Five days later, PBLs were collected and adoptively transferred back into rabbits to induce autoimmune dacryoadenitis.

\section{Clinical and histopathological assessment}

Clinical assessments including tear production, tear break-up time (BUT) and cornea fluorescein staining were performed every two weeks after injection of activated PBLs. Specific inspection method and scoring criteria were the same as those described previously [18]. Rabbits were sacrificed at $6 \mathrm{w}$ and the right inferior LGs were dissected out for the histologic analysis. Tissues were fixated in 10\% formalin, embedded in paraffin vax and sectioned.
The sections were stained routinely with hematoxylin and eosin (H\&E) to estimate inflammatory cell infiltration. The number of focus (aggregate of $>50$ lymphocytes) per $4 \mathrm{~mm}^{2}$ was recorded by two experienced technicians in a blinded fashion $[18,19]$.

\section{Small RNA-sequencing and data analysis}

Total RNA of LGs in the normal and model group rabbits was extracted using the Trizol reagent (Invitrogen, Carlsbad, CA, USA) according to the manufacturer's instruction. The pooled RNA samples from the three rabbits in each group were subjected to Small RNA-sequencing and the Small RNA libraries were generated using the NEBNEXT library generation kit (New England Biolabs, Ltd., USA) following manufacturer's protocols in CapitalBio Corporation (Beijing, China). The library quality was assessed on an Agilent Bioanalyzer 2100 system and sequenced on the Illumina NextSeq Hiseq2500 platform (Illumina, San Diego, CA, USA).

For data analysis, clean reads were obtained by removing reads that containing adaptors and filtering low-quality reads. Then the clean reads were aligned to the reference genome by bowtie [20] and the sequences that matched pre-miRs were considered to be known miRs based on miRBase v22. After standardizing the value of reads by transcripts reads number per million (TPM) [21], the differentially expressed miRs between normal and model group rabbits were screened using the criteria of $|\mathrm{FC}|>1.5$ and false discovery rate $($ FDR $)<0.05$.

\section{qRT-PCR}

$1 \mu \mathrm{g}$ total RNAs were reverse transcribed using specific stem-loop primer. qRT-PCR was performed using FastStart Universal SYBR Green master (ROX) kit (Roche, Germany) on ABI 7900HT Real-Time PCR system (Thermo Fisher Scientific, Waltham, MA, USA). The amplification level was programmed with a denaturation step of $3 \mathrm{~min}$ at $95^{\circ} \mathrm{C}$, followed by 40 cycles of denaturation at $95^{\circ} \mathrm{C}$ for $12 \mathrm{~s}$, and extension at $62{ }^{\circ} \mathrm{C}$ for $40 \mathrm{~s}$. For relative miRs expression level, all data were normalized to the expression level of $\mathrm{U} 6$. Data were calculated using the $2[\Delta \mathrm{Ct}($ Control)- $\Delta \mathrm{Ct}$ (target)] method. Reverse transcription and qRT-PCR primers for miRs and U6 snRNA are listed in Table 1.

\section{miRs target gene enrichment analysis}

The potential target genes of differentially expressed miRs were obtained from miRanda. Then a target genes network map was constructed using Cytoscape software v3.7.0. Functional annotation of potential target genes was performed by GO terms and KEGG pathway enrichment analysis with the threshold of corrected $p$-value $<0.05$. 
Table 1. Primer sequences of stem-loop qRT-PCR

\begin{tabular}{llll}
\hline Name & Primer sequence(5'-3') & \\
\cline { 2 - 4 } & Reverse transcription & Forward \\
\hline ocu-miR-142-3p & GTCGTATCCAGTGCAGGGTCCGAGGTATTCGCACTGGATACGACTCCATA & CGCGCGTAGTGTTTCCTACTT & AGTGCAGGGTCCGAGGTATT \\
ocu-miR-142-5p & GTCGTATCCAGTGCAGGGTCCGAGGTATTCGCACTGGATACGACAGTAGT & GCGCGCATAAAGTAGAAAGC & AGTGCAGGGTCCGAGGTATT \\
ocu-miR-150-5p & GTCGTATCCAGTGCAGGGTCCGAGGTATTCGCACTGGATACGACCACTGG & GCGTCTCCCAACCCTCGTA & AGTGCAGGGTCCGAGGTATT \\
ocu-miR-381-3p & GTCGTATCCAGTGCAGGGTCCGAGGTATTCGCACTGGATACGACACAGAG & CGCGTATACAAGGGCAAGCT & AGTGCAGGGTCCGAGGTATT \\
ocu-miR-31-5p & GTCGTATCCAGTGCAGGGTCCGAGGTATTCGCACTGGATACGACACAGCT & CGAGGCAAGATGCTGGCAT & AGTGCAGGGTCCGAGGTATT \\
ocu-miR-34a-5p & GTCGTATCCAGTGCAGGGTCCGAGGTATTCGCACTGGATACGACACAACC & CGCGTGGCAGTGTCTTAGCT & AGTGCAGGGTCCGAGGTATT \\
U6 & TTCACGAATTTGCGTGTCATC & CGCTTCGGCAGCACATATAC & TTCACGAATTTGCGTGTCATC
\end{tabular}

A
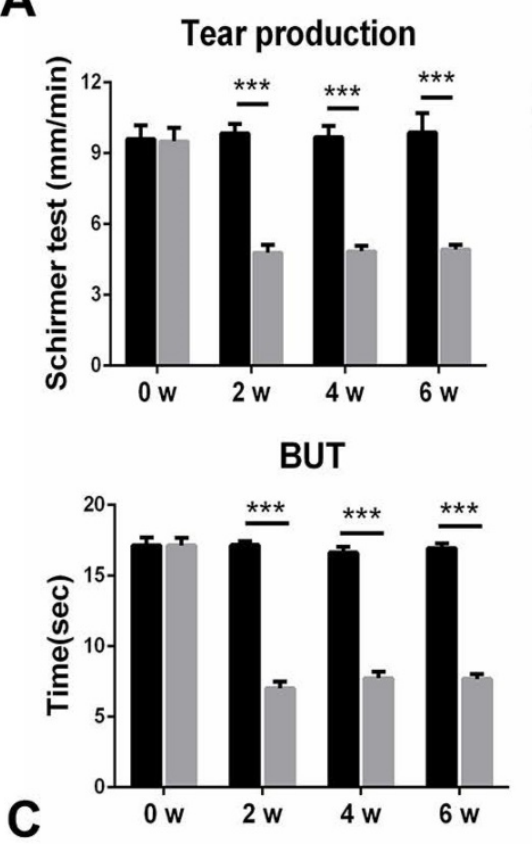

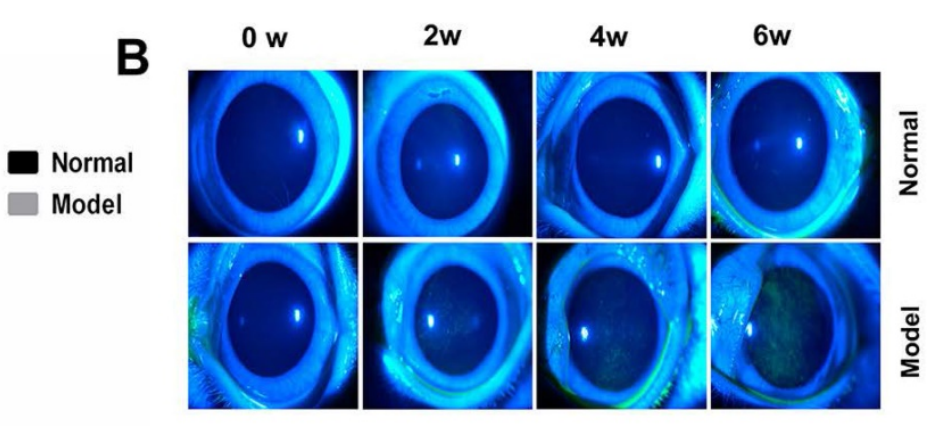

Corneal fluorescein staining

Normal

Model

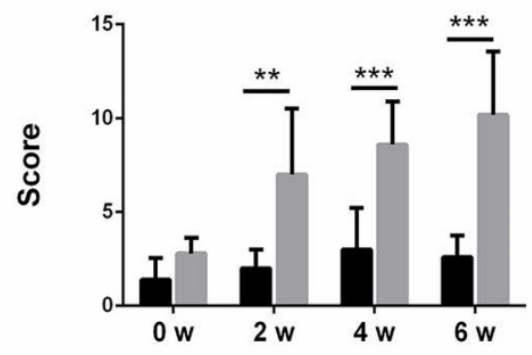

Normal

Model
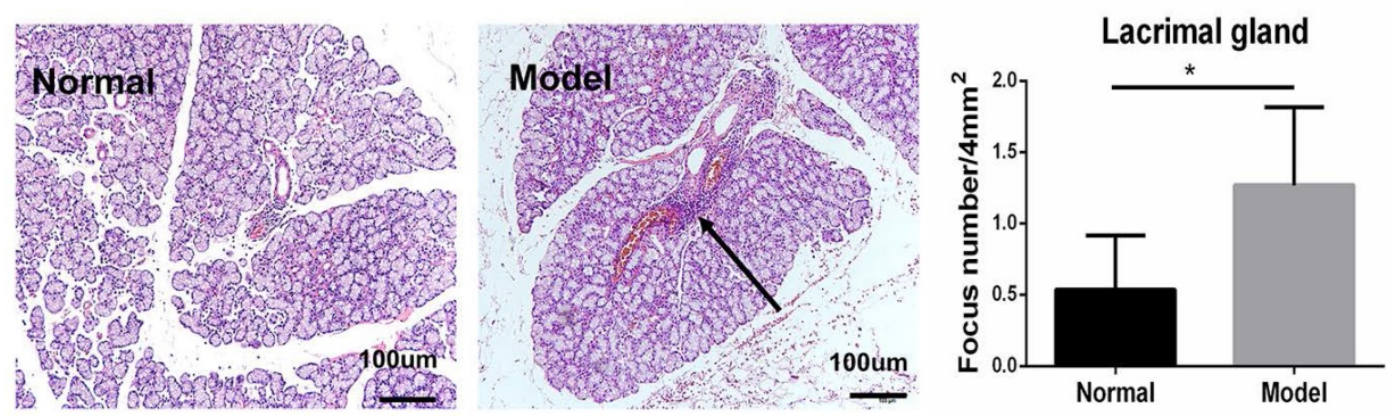

Figure 1. Clinical ocular surface signs and histopathology analysis in rabbit autoimmune dacryoadenitis. (A) The clinical scores of rabbits were assessed every 2 weeks in a blinded fashion after adoptive transfer of activated PBLs. (B) Representative photographs of fluorescein staining in model and normal rabbits. (C) Histologic analysis of LGs from normal and model group rabbits. Arrows shows perivascular or periductal lymphocytic foci. $n=5$ rabbits per group. Model group versus Normal group, ${ }^{*} p<0.05$, $* * p<0.01$ or $* * * p<0.001$.

\section{Statistical analysis}

Data were analyzed with Graphpad prism 6.02 and presented as mean \pm SD. The normality of the data was evaluated using Shapiro-Wilk test. When normality was not rejected, comparisons were made using Student's $t$ test or two-way analysis of variance (ANOVA). $P<0.05$ is considered to be statistically significant.

\section{Results}

\section{Clinical and histological examination of} autoimmune dacryoadenitis

Autoimmune dacryoadenitis was induced in rabbits by injection of activated autologous PBLs via ear margin vein. Clinical assessments were performed every 2 weeks and the histopathological examination was performed on week 6 after adoptive transfer $(n=5$ /group). As shown in Figure $1 A-B$, the model 
group developed severe dry eye syndrome, characterized by decreased tear production, shortened tear BUT, and abnormal corneal epithelium staining, relative to the normal controls. Consistent with the clinical results, histological analysis of LGs revealed that inflammatory cell infiltration increased significantly in model group rabbits compared to the normal controls (Figure 1C).

\section{Identification of differential miRs profiles}

To identify the difference of miRs expression in autoimmune dacryoadenitis, we employed small RNA-sequencing to analyze miRs profiles of LGs in model and normal rabbits. The results showed that the expression levels of 249 out of 367 miRs were modulated in LGs of autoimmune dacryoadenitis rabbits relative to normal ones. Using the threshold values $\log 2 \mathrm{FC}>1.5$ or $\log 2 \mathrm{FC}<-1.5$, and $\mathrm{P}<0.05$, we identified 15 differentially expressed miRs including 9 upregulated (miR-150-5p, miR-142-3p, miR-142-5p, miR-335-3p, miR-144-3p, miR-188-5p, miR-671-5p, miR-378-5p, miR-653-5p) and 6 downregulated miRs (miR-34a-5p, miR-31-5p, miR-381-3p, miR-219a-5p, miR-656-3p, miR-432-5p) (Figure 2A).

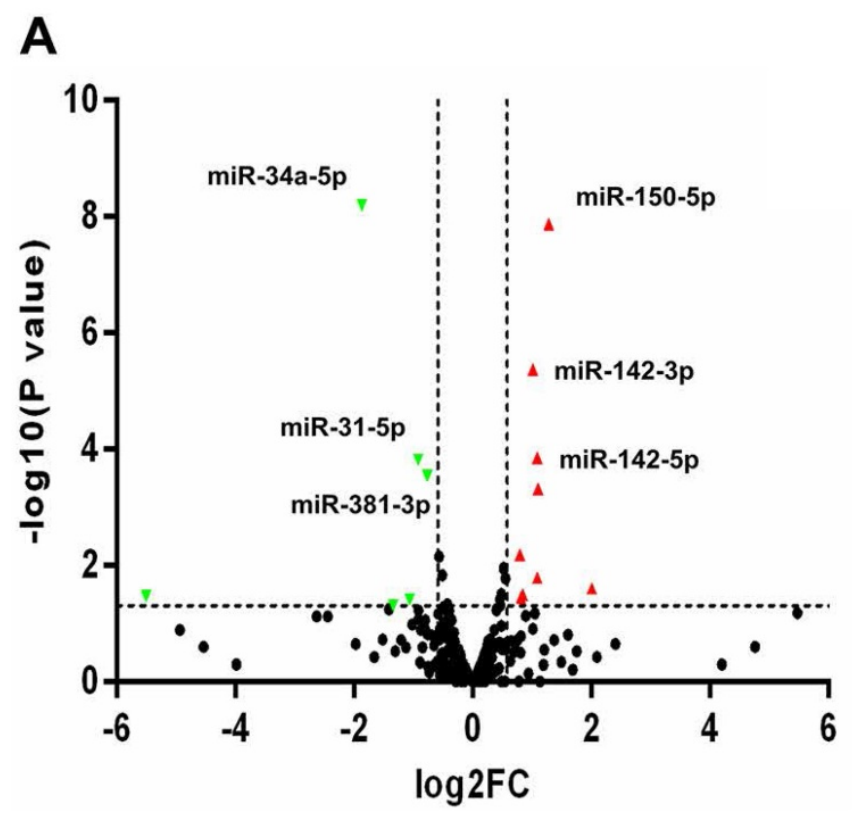

B
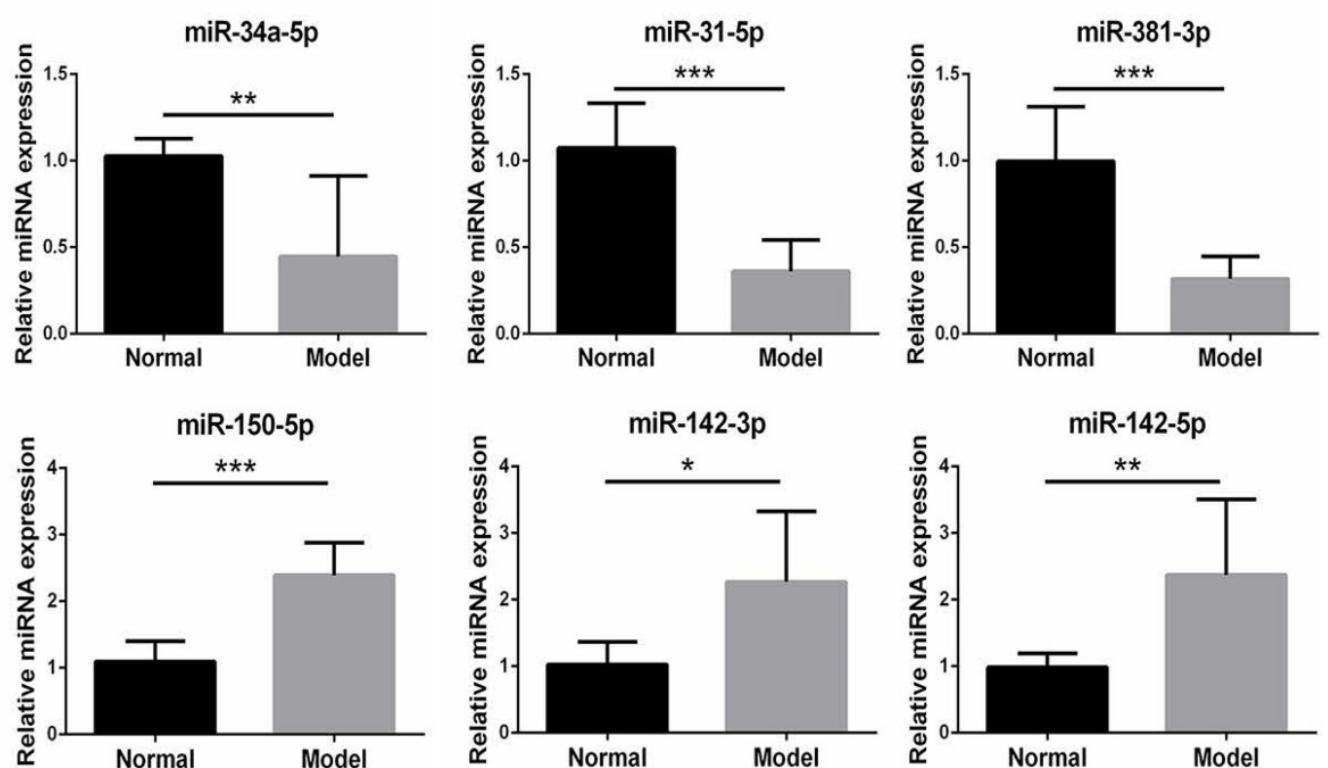

Figure 2. (A) The "volcano plot" picture of the differentially expressed miRs between normal and model group rabbits. Red triangles indicate upregulated miRs and green triangles represent downregulated miRs. (B) Validation of the expression patterns of the selected miRs using qRT-PCR. The relative expression of each miRs was normalized to U6 snRNA. The data shown are representative of at least three independent experiments and represented as mean \pm SD. Statistically significant between normal and model group rabbits is indicated by $* p<0.05, * * p<0.01$ or $* * * p<0.001$. 

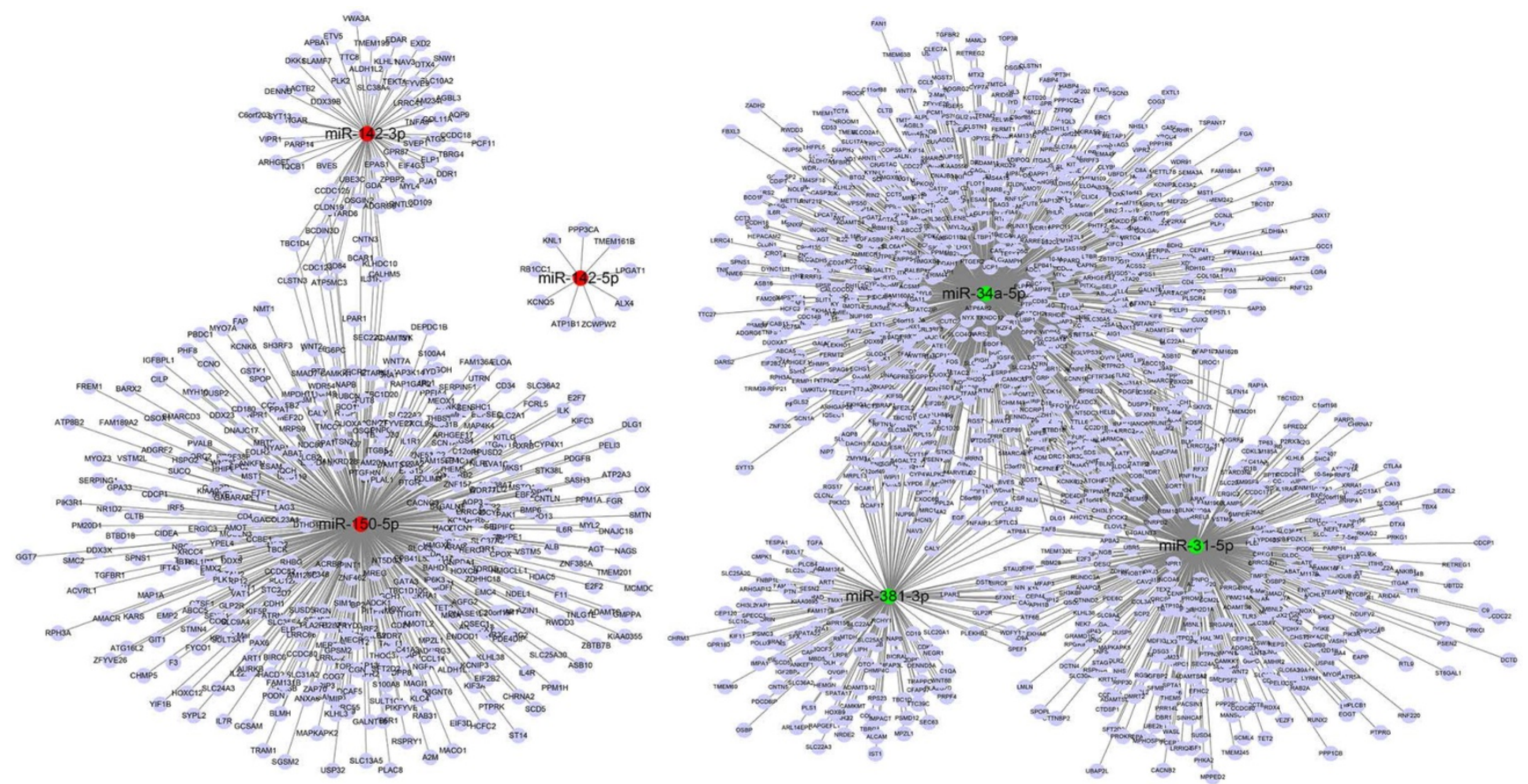

Figure 3. miRs-target genes networks of six most significantly differentially expressed miRs. Red indicates upregulated miRs, green indicates downregulated miRs, purple represents target genes.

\section{Validation of differentially expressed miRs with qRT-PCR}

To confirm the small RNA-seq results, three upregulated miRs and three downregulated miRs among the filtered ones were validated with qRT-PCR between the model rabbits and normal samples $(n=3$ /group). As shown in Figure 2B, the expression of miR-34a-5p, miR-381-3p and miR-31-5p was significantly decreased in model group rabbits compared with normal ones, whereas the miR-150-5p, miR-142-3p and miR-142-5p showed an up-regulated expression pattern, which was in agreement with the small RNA-seq results.

\section{Prediction of target mRNAs for differential expression miRs}

The target genes of 15 differentially expressed miRs in rabbit autoimmune dacryoadenitis were predicted using miRanda database, and a total of 2215 target genes were obtained. As shown in Figure 3, 524 mRNAs were predicted as targets of miR-31-5p, 191 mRNAs were predicted as targets of miR-381-3p and 959 mRNAs were predicted as targets of miR-34a-5p. Moreover, both miR-31-5p and miR-381-3p target TNFAIP1, a protein that is derived from TNF and plays a key role in MAPK signaling pathway [22]. Meanwhile, 513 genes were predicted as targets of miR-150-5p, in which MAPKAPK2 is activated by MAPK p38 and has been proved to be a therapeutic target for inhibition of chronic inflammatory diseases
[23]. Additionally, 77 genes were predicted as targets of miR-142-3p and 9 mRNAs were predicted as targets of miR-142-5p.

\section{GO enrichment analysis}

GO enrichment analysis was performed to analyze the target gene functions. The top $30 \mathrm{GO}$ terms in three categories are shown in Figure 4A. On the basis of biological process (BP), the top three processes were $G$ protein-coupled receptor signaling pathway, sensory perception and transport. In molecular function (MF), target genes were significantly enriched in transmembrane signaling receptor activity. As for cellular component (CC), the top three processes were intracellular, intracellular part and plasma membrane part. To further understand the role of these altered miRs involved in the development of autoimmune dacryoadenitis, target genes were performed and mapped for GO category: immune system process. As shown in Figure $4 \mathrm{~B}$, the target genes of the differentially expressed miRs were significantly enriched in Toll-like receptor signaling pathway, regulation of immune response and $\mathrm{T}$ cell activation and proliferation.

\section{KEGG pathway analysis}

KEGG pathway analysis showed the top twenty signaling pathways enriched with the target genes of the differentially expressed miRs (Figure 5A). The target genes in the model group rabbits were mainly enriched in MAPK signaling pathway, Focal 
adhesion, and NF-kB signaling pathway. We focused our attention on the MAPK signaling pathway, which is the most significant enrichment pathway and has been reported to be involved in the pathogenesis of SS dry eye. Remarkably, 18 genes at different level of MAPK signaling pathway were targeted by four identified downregulated miRs (Figure 5B).

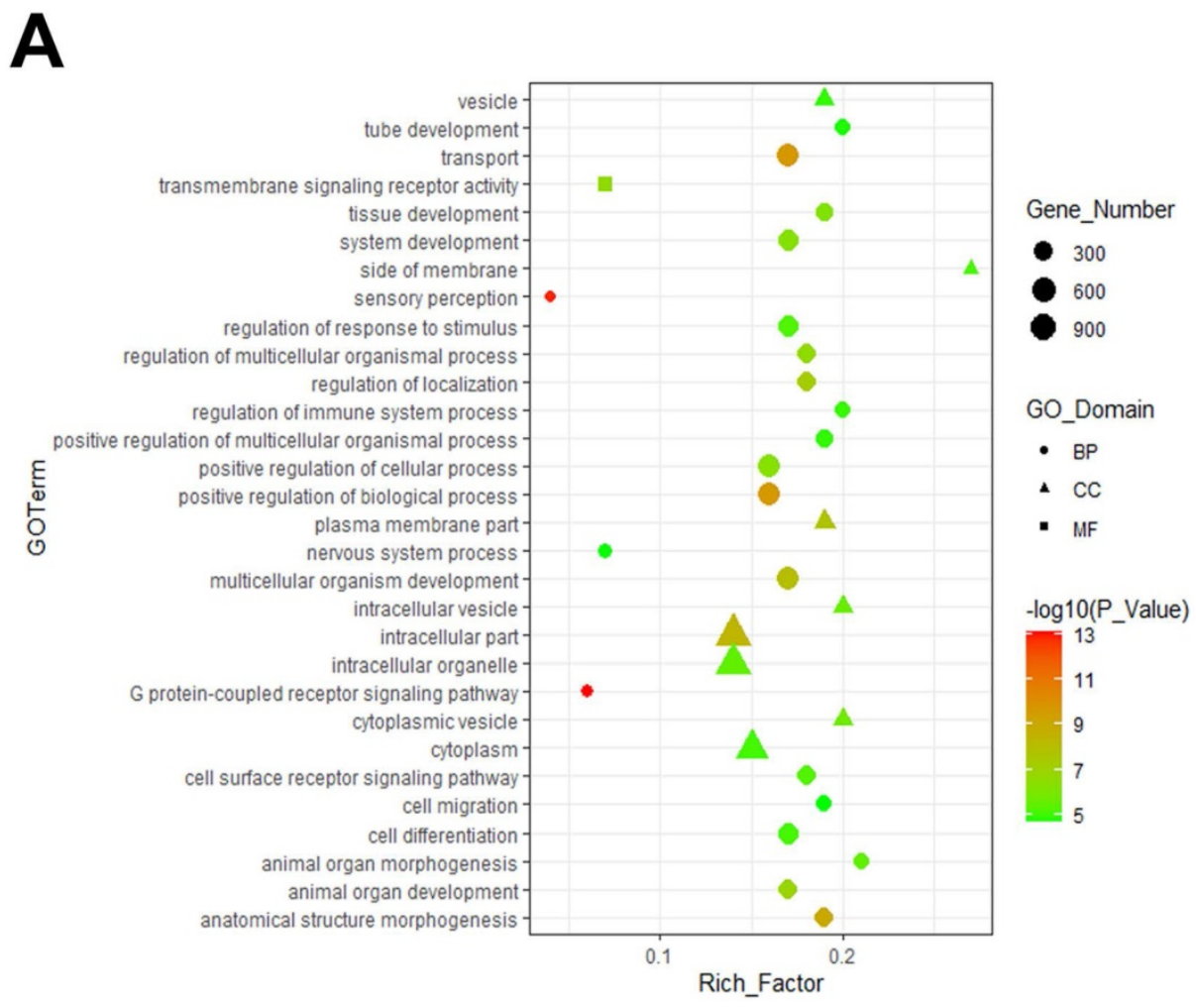

B

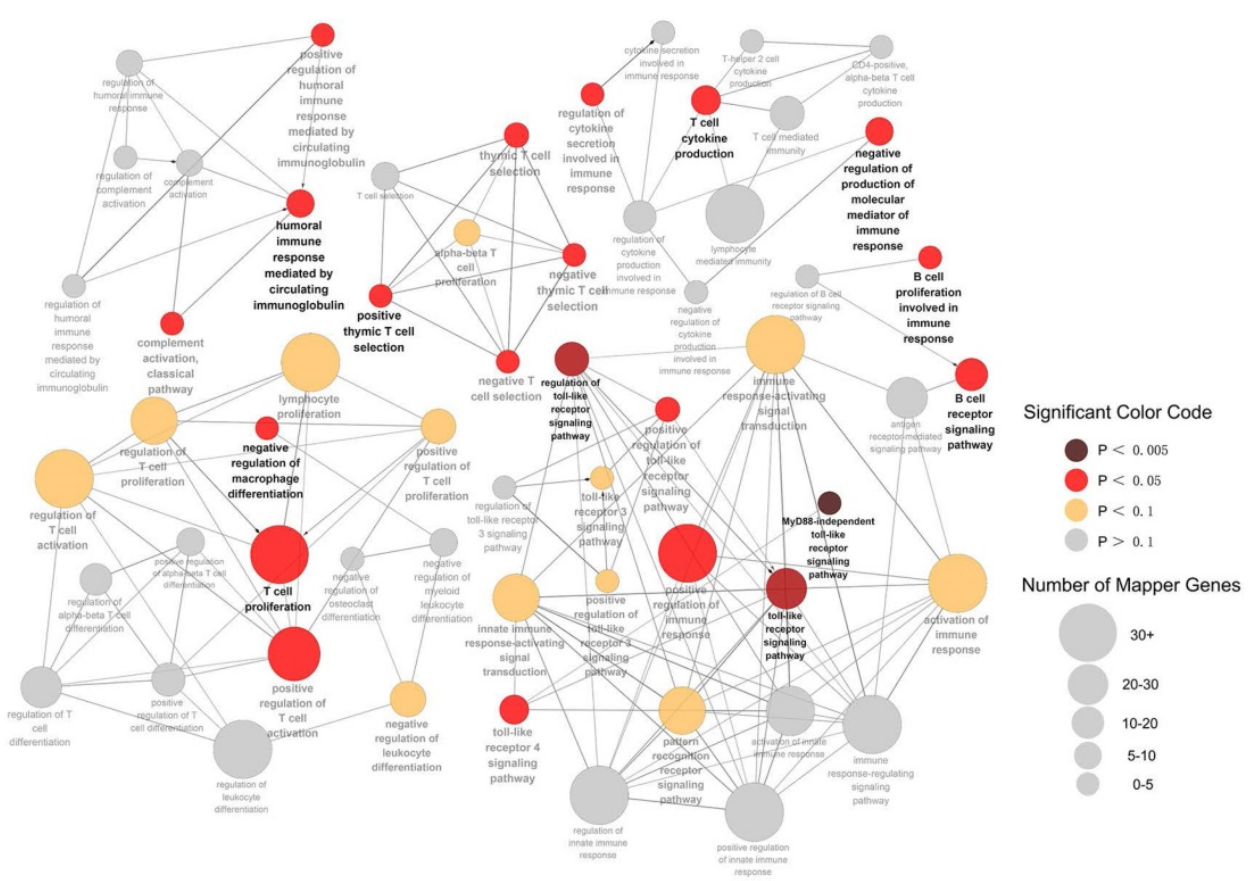

Figure 4. GO terms enrichment analysis. (A) GO analysis of predicted genes was performed in accordance with the BP, CC and MF. Shape represents GO categories, color corresponds to the -log10 of $\mathrm{P}$ value, size represents the enrichment gene number. (B) Predicted target genes were mapped for GO category: immune system process. Color represents the $P$ value, and the diameter of each circle represents the number of genes enrichment. 

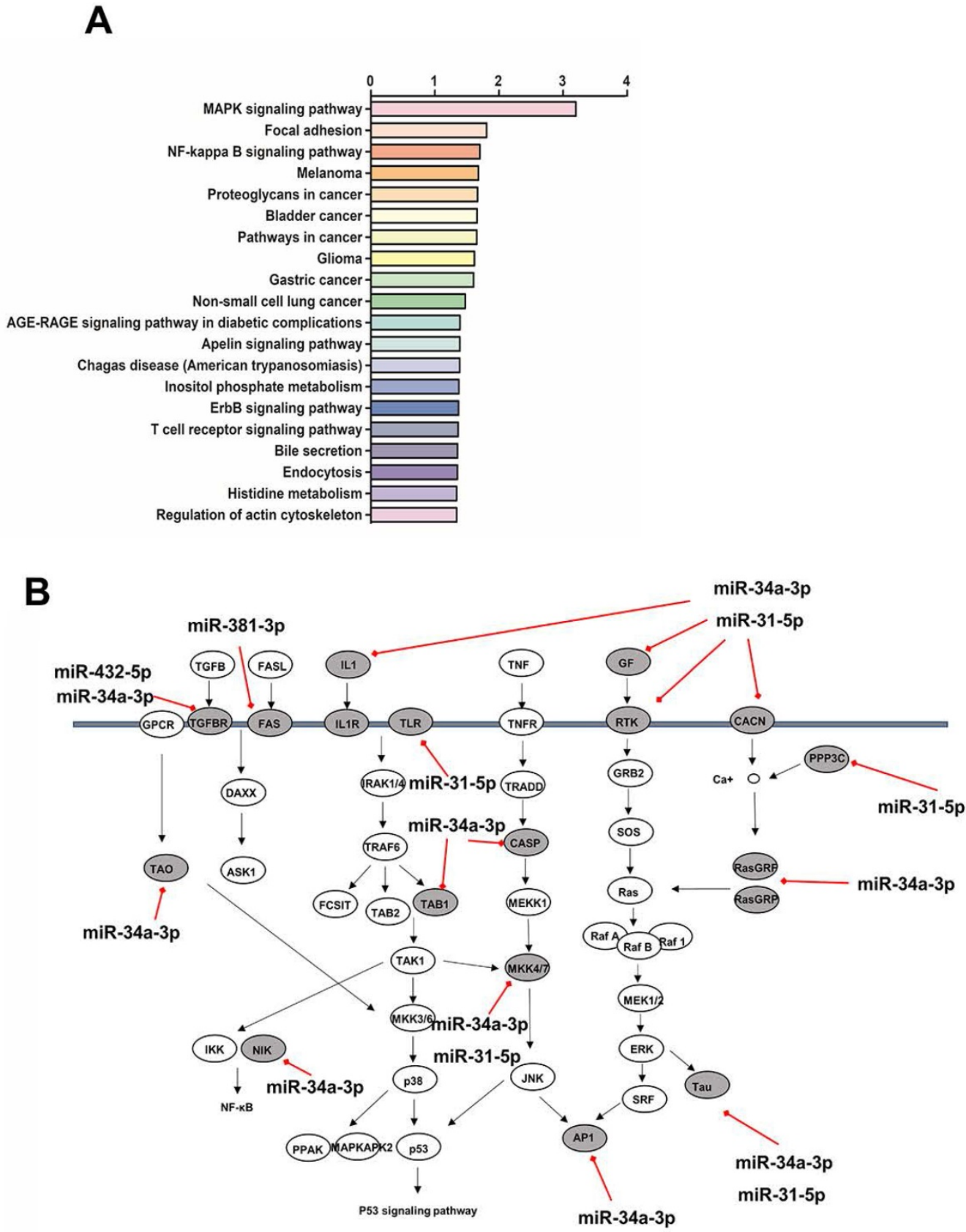

Figure 5. (A) Pathway analysis on target genes of differentially expressed miRs. The top 20 pathways are summarized. (B) miRs downregulated in autoimmune dacryoadenitis target multiple genes in the MAPK signaling pathway. The direct targets of downregulated miRs in the pathway are denoted with gray ovals.

\section{Discussion}

SS dry eye is a multifactorial disease, including genetic predisposition, environmental and epigenetic factors [12]. Recently, accumulating evidences have revealed that miRs are dysregulated in tears, PBMCs, and salivary gland of patients with SS [15, 17, 24]. Nevertheless, the miRs profiling in LGs of SS dry eye remains unclear. In the present study, we performed small RNA-seq followed by qRT-PCR validation, and functional analysis to explore their role in autoimmune dacryoadenitis. Bioinformatics analysis revealed that some specific miRs might participate in the development of autoimmune dacryoadenitis via modulation of MAPK signaling pathway, Focal adhesion, and NF-KB signaling pathway.

In this study, we identified 15 differentially expressed miRs in LGs of autoimmune dacryoadenitis rabbits. Several of them have been reported to be involved in other inflammatory eye diseases. Guo et al. revealed that miR-150 and miR-653 were upregulated in peripheral blood lymphocytes in rats with experimental autoimmune uveitis [25]. As for SS, previous studies reported that miR-335 was upregulated in the minor salivary gland of SS patients, and its expression level was inversely 
proportional to the unstimulated salivary flow rate [26]. In addition, Johansson et al. identified miR-31 was reduced in the T cells of SS patients, which supports the autoimmune $\mathrm{T}$ cell response during chronic type I IFN exposure [27]. These findings were consistent with our results that miR-335-3p was upregulated, whereas miR-31-5p was downregulated in autoimmune dacryoadenitis rabbits compared to the controls, suggesting these miRs may play a potential role in the pathogenesis of autoimmune dry eye. The identification of LGs specific miRs in our results may contribute to the pathogenesis of LGs dysfunction in SS dry eye.

To explore how these differentially expressed miRs participate in autoimmune dacryoadenitis pathogenesis, a miRs-mRNAs network was generated, which identified the potential link between miRs and their downstream targets. In this study, we note that the target genes of the significantly upregulated miRs, such as TGF $\beta 2$ and NFE2L2, may be closely associated with the pathogenesis of autoimmune dry eye. TGF $\beta 2$ is a crucial mediator of the ocular inflammation in dry eye disease [28]. Besides, Stephen et al. found that the therapeutic effect of the cyclosporine emulsion on the chronic phase of autoimmune dry eye might be attributed to the increased expression of TGF $\beta 2$ [29]. NFE2L2 has previously been reported to affect cell migration and promote the corneal epithelial wound-healing process [30]. Most recently, Matsuda et al. found RS9, a novel NFE2L2 activator, can induce the expression of NFE2L2-targeted genes, including NQO1 and GCLC, and ameliorate the symptoms of dry eye, implying the important role of NFE2L2 in the development of dry eye disease [31]. With regards to the target gene of downregulated miRs, abnormal activation of P2RX7 has been revealed to participate in the development of autoimmune dry eye by inducing severe LGs dysfunction, and inhibition of P2RX7 may represent a promising therapeutic strategy for the management of autoimmune dry eye [32, 33]. Therefore, we concluded that differentially expressed miRs may regulate the pathogenesis of autoimmune dacryoadenitis by modulating these target genes expression.

Our bioinformatics analysis showed that target genes of the differentially expressed miRs mainly enriched in MAPK signaling pathway. The MAPK pathways, including p38, extracellular signal regulated kinases (ERK) and c-jun $\mathrm{N}$-terminal kinases (JNK), are pivotal regulators of various cellular activities, such as inflammation and innate immunity [34]. Furthermore, MAPK signaling pathways have been reported to be critical for the production of inflammatory cytokines IL-1 $\beta$ and TNF- $\alpha$, and matrix metalloproteinase (MMPs), which are involved in the pathogenesis of dry eye [34,35]. More importantly, Zoukhri et al. found the activation of JNK participated in IL-1 $\beta$-induced impaired tear secretion in inflamed LGs [36]. Besides, MAPK inhibitors such as SP600125 and SB203580 could ameliorate dry eye associated symptoms and reduce inflammatory cytokines production in tears of experimental dry eye disease, suggesting that inhibiting the activation of MAPK signaling pathway is beneficial to the treatment of dry eye [36, 37]. Our results suggest that the downregulated miRs may participate in the pathogenesis of autoimmune dry eye by activating MAPK pathways.

The present study has several limitations. First, the number of samples was relatively small; and second, the target genes and signaling pathways of differentially expressed miRs were obtained from existing databases. These findings should be confirmed by further mechanistic studies. Additionally, the correlation between the degree of inflammation of autoimmune dry eye and miRs expression level could not be accurately investigated.

Taken together, our study identified changes in the expression of miRs in LGs of rabbit autoimmune dacryoadenitis and suggested the potential roles of these differentially expressed miRs in the pathogenesis of autoimmune dry eye. The present study here may provide important clues to the development of potential miRs-based therapeutic strategies for human autoimmune dry eye disease.

\section{Abbreviations}

miRs: microRNAs; LGs: lacrimal glands; PBLs: peripheral blood lymphocytes; qRT-PCR: quantitative real time-polymerase chain reaction; GO: Gene Ontology; KEGG: Kyoto Encyclopedia of Genes and Genomes; SS: Sjögren's syndrome; PBMCs: peripheral blood mononuclear cells; pLGECs: purified LG epithelial cells; BUT: tear break-up time; MF: molecular function; CC: cellular component; BP: biological process.

\section{Acknowledgements}

This work was supported by the National Natural Science Foundation of China (81970793, 81770901), and the Tianjin Clinical Key Discipline Project (TJLCZDXKT003).

\section{Competing Interests}

The authors have declared that no competing interest exists.

\section{References}

1. Zang S, Cui Y, Cui Y, Fei W. Meibomian gland dropout in Sjogren's syndrome and non-Sjogren's dry eye patients. Eye (Lond). 2018; 32: 1681-7. 
2. Ogawa Y, Shimizu E, Tsubota K. Interferons and Dry Eye in Sjogren's Syndrome. International journal of molecular sciences. 2018; 19: 3548.

3. Foulks GN, Forstot SL, Donshik PC, Forstot JZ, Goldstein MH, Lemp MA, et al. Clinical guidelines for management of dry eye associated with Sjogren disease. Ocul Surf. 2015; 13: 118-32.

4. Wei Y, Chen S, Sun D, Li X, Wei R, Li X, et al. miR-223-3p promotes autoreactive Th17 cell responses in experimental autoimmune uveitis (EAU) by inhibiting transcription factor FOXO3 expression. FASEB journal : official publication of the Federation of American Societies for Experimental Biology. 2019; 33: 13951-65.

5. Yan B, Yao J, Liu JY, Li XM, Wang XQ, Li YJ, et al. IncRNA-MIAT regulates microvascular dysfunction by functioning as a competing endogenous RNA. Circulation research. 2015; 116: 1143-56.

6. Xiao F, Han M, Wang X, Gong X, Huang E, Zhu Z, et al. Animal models of Sjogren's syndrome: an update. Clin Exp Rheumatol. 2019; 37 Suppl 118: 209-16.

7. Guo Z, Song D, Azzarolo AM, Schechter JE, Warren DW, Wood RL, et al. Autologous lacrimal-lymphoid mixed-cell reactions induce dacryoadenitis in rabbits. Experimental eye research. 2000; 71: 23-31.

8. Thomas PB, Zhu Z, Selvam S, Samant DM, Stevenson D, Mircheff AK, et al. Autoimmune dacryoadenitis and keratoconjunctivitis induced in rabbits by subcutaneous injection of autologous lymphocytes activated ex vivo against lacrimal antigens. Journal of autoimmunity. 2008; 31: 116-22.

9. Lu TX, Rothenberg ME. MicroRNA. The Journal of allergy and clinical immunology. 2018; 141: 1202-7.

10. Bernardo BC, Ooi JY, Lin RC, McMullen JR. miRNA therapeutics: a new class of drugs with potential therapeutic applications in the heart. Future medicinal chemistry. 2015; 7: 1771-92.

11. Tu Y, Guo R, Li J, Wang S, Leng L, Deng J, et al. MiRNA Regulation of MIF in SLE and Attenuation of Murine Lupus Nephritis With miR-654. Frontiers in immunology. 2019; 10: 2229.

12. Reale M, D'Angelo C, Costantini E, Laus M, Moretti A, Croce A. MicroRNA in Sjogren's Syndrome: Their Potential Roles in Pathogenesis and Diagnosis. Journal of immunology research. 2018; 2018: 7510174.

13. Cha S, Mona M, Lee KE, Kim DH, Han K. MicroRNAs in Autoimmune Sjogren's Syndrome. Genomics Inform. 2018; 16: e19.

14. Kapsogeorgou EK, Gourzi VC, Manoussakis MN, Moutsopoulos HM, Tzioufas AG. Cellular microRNAs (miRNAs) and Sjogren's syndrome: candidate regulators of autoimmune response and autoantigen expression. Journal of autoimmunity. 2011; 37: 129-35.

15. Kim YJ, Yeon Y, Lee WJ, Shin YU, Cho H, Sung YK, et al. Comparison of MicroRNA Expression in Tears of Normal Subjects and Sjogren Syndrome Patients. Invest Ophthalmol Vis Sci. 2019; 60: 4889-95.

16. Shi $\mathrm{H}$, Zheng LY, Zhang $\mathrm{P}, \mathrm{Yu}$ CQ. miR-146a and miR-155 expression in PBMCs from patients with Sjogren's syndrome. Journal of oral pathology \& medicine : official publication of the International Association of Oral Pathologists and the American Academy of Oral Pathology. 2014; 43: 792-7.

17. Rassi DM, De Paiva CS, Dias LC, Modulo CM, Adriano L, Fantucci MZ, et al. Review: MicroRNAS in ocular surface and dry eye diseases. Ocul Surf. 2017; 15: 660-9.

18. Li X, Lu X, Sun D, Wang X, Yang L, Zhao S, et al. Adipose-Derived Mesenchymal Stem Cells Reduce Lymphocytic Infiltration in a Rabbit Model of Induced Autoimmune Dacryoadenitis. Invest Ophthalmol Vis Sci. 2016; 57: 5161-70.

19. $\mathrm{Lu} \mathrm{X}, \mathrm{Li} \mathrm{N}$, Zhao $\mathrm{L}, \mathrm{Guo} \mathrm{D}, \mathrm{Yi} \mathrm{H}$, Yang $\mathrm{L}$ et al. Human umbilical cord mesenchymal stem cells alleviate ongoing autoimmune dacryoadenitis in rabbits via polarizing macrophages into an anti-inflammatory phenotype. Experimental eye research. 2020; 191: 107905.

20. Langmead B, Trapnell C, Pop M, Salzberg SL. Ultrafast and memory-efficient alignment of short DNA sequences to the human genome. Genome Biol. 2009; 10: R25.

21. Zhou L, Chen J, Li Z, Li X, Hu X, Huang Y, et al. Integrated profiling of microRNAs and mRNAs: microRNAs located on Xq27.3 associate with clear cell renal cell carcinoma. PLoS One. 2010; 5: e15224.

22. McCarthy PC, Phair IR, Greger $C$, Pardali $K$, McGuire VA, Clark AR, et al. IL-33 regulates cytokine production and neutrophil recruitment via the p38 MAPK-activated kinases MK2/3. Immunology and cell biology. 2019; 97: 54-71.

23. Singh RK, Najmi AK, Dastidar SG. Biological functions and role of mitogen-activated protein kinase activated protein kinase 2 (MK2) in inflammatory diseases. Pharmacological reports : PR. 2017; 69: 746-56.

24. Williams AE, Choi $\mathrm{K}$, Chan AL, Lee YJ, Reeves WH, Bubb MR, et al. Sjogren's syndrome-associated microRNAs in CD14(+) monocytes unveils targeted TGFbeta signaling. Arthritis research \& therapy. 2016; 18: 95.

25. Guo D, Li J, Liu Z, Tang K, Song H, Bi H. Characterization of microRNA expression profiling in peripheral blood lymphocytes in rats with experimental autoimmune uveitis. Inflammation research : official journal of the European Histamine Research Society. 2015; 64: 683-96.

26. Martini D, Gallo A, Vella S, Sernissi F, Cecchettini A, Luciano N, et al. Cystatin S-a candidate biomarker for severity of submandibular gland involvement in Sjogren's syndrome. Rheumatology (Oxford, England). 2017; 56: 1031-8.

27. Johansson A, Nyberg WA, Sjostrand M, Moruzzi N, Bergman P, Khademi M, et al. miR-31 regulates energy metabolism and is suppressed in T cells from patients with Sjogren's syndrome. European journal of immunology. 2019; 49: $313-22$.
28. Benito MJ, Calder V, Corrales RM, Garcia-Vazquez C, Narayanan S, Herreras JM, et al. Effect of TGF-beta on ocular surface epithelial cells. Experimental eye research. 2013; 107: 88-100.

29. Pflugfelder SC, De Paiva CS, Villarreal AL, Stern ME. Effects of sequential artificial tear and cyclosporine emulsion therapy on conjunctival goblet cell density and transforming growth factor-beta2 production. Cornea. 2008; 27: 64-9.

30. Hayashi R, Himori N, Taguchi K, Ishikawa $\mathrm{Y}$, Uesugi K, Ito M, et al. The role of the Nrf2-mediated defense system in corneal epithelial wound healing. Free radical biology \& medicine. 2013; 61: 333-42.

31. Matsuda Y, Machida M, Nakagami Y, Nakajima T, Azuma M. NFE2L2 activator RS9 protects against corneal epithelial cell damage in dry eye models. PLoS One. 2020; 15: e0229421.

32. Yu J, Chen $\mathrm{Y}$, Li M, Gao Q, Peng Y, Gong Q, et al. Paeoniflorin down-regulates ATP-induced inflammatory cytokine production and P2X7R expression on peripheral blood mononuclear cells from patients with primary Sjogren's syndrome. International immunopharmacology. 2015; 28: 115-20.

33. Khalafalla MG, Woods LT, Camden JM, Khan AA, Limesand KH, Petris MI, et al. P2X7 receptor antagonism prevents IL-1beta release from salivary epithelial cells and reduces inflammation in a mouse model of autoimmune exocrinopathy. The Journal of biological chemistry. 2017; 292: 16626-37.

34. Luo L, Li DQ, Doshi A, Farley W, Corrales RM, Pflugfelder SC. Experimental dry eye stimulates production of inflammatory cytokines and MMP-9 and activates MAPK signaling pathways on the ocular surface. Invest Ophthalmol Vis Sci. 2004; 45: 4293-301.

35. De Paiva CS, Corrales RM, Villarreal AL, Farley WJ, Li DQ, Stern ME, et al. Corticosteroid and doxycycline suppress MMP-9 and inflammatory cytokine expression, MAPK activation in the corneal epithelium in experimental dry eye. Experimental eye research. 2006; 83: 526-35.

36. Zoukhri D, Macari E, Choi SH, Kublin CL. c-Jun NH2-terminal kinase mediates interleukin-1beta-induced inhibition of lacrimal gland secretion. Journal of neurochemistry. 2006; 96: 126-35.

37. Ma X, Zou J, He L, Zhang Y. Dry eye management in a Sjogren's syndrome mouse model by inhibition of p38-MAPK pathway. Diagnostic pathology. 2014; 9: 5 . 